\title{
Experimental Investigation of Effect on Hydrate Formation in Spray Reactor
}

\author{
Jianzhong Zhao, ${ }^{1}$ Yaqin Tian, ${ }^{2}$ Yangsheng Zhao, ${ }^{1}$ and Wenping Cheng ${ }^{3}$ \\ ${ }^{1}$ Mining Technology Institute, Taiyuan University of Technology, Taiyuan 030024, China \\ ${ }^{2}$ College of Materials Science and Engineering, Taiyuan University of Science and Technology, Taiyuan 030024, China \\ ${ }^{3}$ College of Chemistry and Chemical Engineering, Taiyuan University of Technology, Taiyuan 030024, China
}

Correspondence should be addressed to Jianzhong Zhao; zjz1104@163.com

Received 27 July 2015; Accepted 28 September 2015

Academic Editor: Tomokazu Yoshimura

Copyright (C) 2015 Jianzhong Zhao et al. This is an open access article distributed under the Creative Commons Attribution License, which permits unrestricted use, distribution, and reproduction in any medium, provided the original work is properly cited.

\begin{abstract}
The effects of reaction condition on hydrate formation were conducted in spray reactor. The temperature, pressure, and gas volume of reaction on hydrate formation were measured in pure water and SDS solutions at different temperature and pressure with a high-pressure experimental rig for hydrate formation. The experimental data and result reveal that additives could improve the hydrate formation rate and gas storage capacity. Temperature and pressure can restrict the hydrate formation. Lower temperature and higher pressure can promote hydrate formation, but they can increase production cost. So these factors should be considered synthetically. The investigation will promote the advance of gas storage technology in hydrates.
\end{abstract}

\section{Introduction}

Gas hydrates (clathrate hydrates) are crystalline solid structures consisting of water and small molecules such as $\mathrm{CO}_{2}$, $\mathrm{N}_{2}, \mathrm{CH}_{4}$, and $\mathrm{H}_{2}$, which are formed under certain thermodynamic conditions [1]. Gas hydrate is a case-like structure made of hydrogen-bonded water molecules. This structure is stabilized with including gas molecule. Since gas hydrate can take in various gas molecules, several applications have been proposed in environmental and energy fields, for example, natural gas or coal bed gas storage and transportation in the form of gas hydrates [2-4], separation processes of gaseous mixtures using differences in hydrate formation tendencies among the gaseous components [5, 6], and ocean disposal of anthropogenic $\mathrm{CO}_{2}$ as a mitigation measure for global warming. Most of these applications dispose a lot of gas hydrate. For the implementation of these applications, development of efficient processes of gas hydrate formation would be necessary. Efficient and fast generation of gas hydrate is a key factor for the storage and separation of gas hydrate $[7,8]$. The formation of hydrate is controlled by many factors, such as gas composition, reaction temperature and pressure, contact area of water and gas, and additives $[9,10]$. These factors affect the formation of gas hydrate, the reaction rate, and the content of gas [11]. From the aspects of the process of crystallization, the formation of gas hydrate consists of three stages: dissolution, nucleation, and crystal growth. Nucleation process is a key factor to control the growth of gas hydrate. Once the crystal nucleus formatted hydrate can generate rapidly in the critical nuclei. Influence of additives, temperature, and pressure macroscopic thermodynamic factors on hydrate formation process is analyzed by experiment. The study reveals the patterns, the influence of these factors on the hydrate formation rate, gas rate and reaction driving force, and other physical and chemical indicators.

\section{Experiment}

2.1. Materials. $\mathrm{CH}_{4}$ (99.99\%) was supplied by Beijing AP BAIF Gases Industry Co., Ltd., used for hydrate formation with distilled water and additives. Purity of sodium dodecyl sulfate (SDS) is $99 \%$. Ethylene Glycol also supplied by Nanjing Chemicals was used as coolant. SDS solutions were prepared at $300 \mathrm{ppm}(\mathrm{mg} / \mathrm{kg})$. All chemicals were used as received. All other chemicals were used without further purification. 


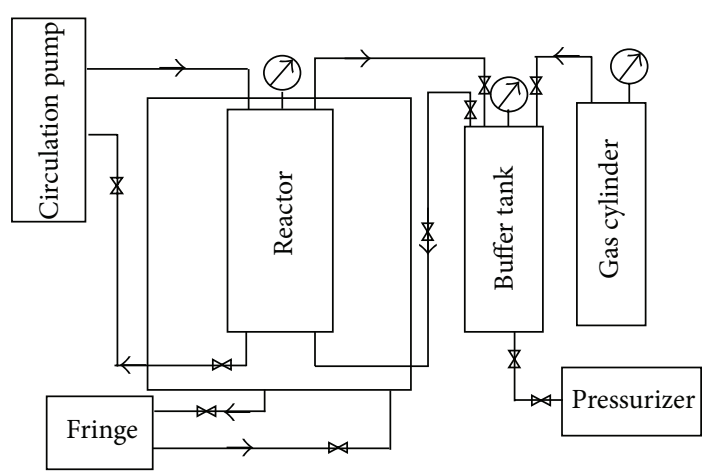

FIGURE 1: Experiment flow of hydrate formation.

2.2. Experimental Procedure. First of all, the experimental gas was injected into the reactor with a water filled tank until the remaining volume of the reaction is required. Then the gas supply booster pump is started, and the freezing unit starts to cool the reactor. When the pressure and temperature are reached, the circulating pump is opened to the reaction process. As the reaction is carried out, the consumption volume of the gas is automatically added to the booster pump to ensure that the pressure is constant during the reaction. When the pressure and temperature are constant, the pressure relief operation can be carried out. Finally, the formated hydrate was taken out and the gas content rate and other physical and chemical indicators were determinated. In the experiment data is automatically detected and recorded by the control display system. The experimental procedure is shown in Figure 1.

2.3. Analyses of Experimental Processes. At the beginning of the reaction, the temperature and the pressure change slowly, and the reaction is in the induction period. According to the crystal growth theory, this period is a nuclear phase. The nucleation of hydrates can be defined as a process of formation of a critical size and stable nucleation which is formed in the solution of the hydrate forming gas [12]. When the solution is in a cold state or in a saturated state, nucleation can occur. In the nucleation stage, the water molecules cluster changes the original hydrogen bond network structure and the gas molecules rearrange into the gas water molecules. Under the suitable physical condition, the gas water molecular cluster grows into a cage-like structure, and the critical crystal nucleus is formed. With the reaction, the pressure and temperature increase. With the increase of gas consumption, the work time of gas booster pump is also increased. At the same time, the temperature of the reactor also has a certain increase. Reaction enters the crystal growth stage. The process of crystal growth is a process of interface. The essence of the crystal growth process is the migration of the solute molecules on the surface of the crystal. The process of macro performance is the formation of a large amount of gas hydrate $[13,14]$.

\section{Discussions}

The effects of active agents, pressure, and temperature on the formation rate and the final gas content of the hydrate

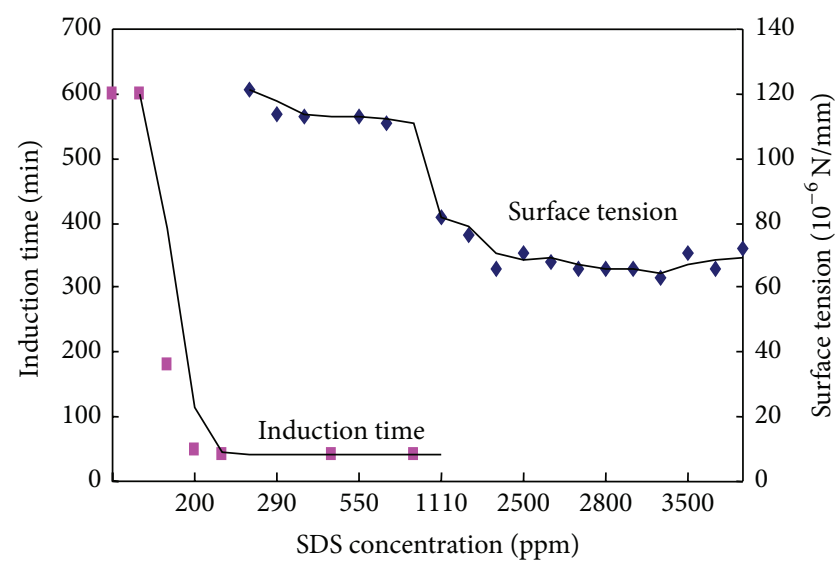

FIGURE 2: Induction time and surface tension versus SDS concentration.

were studied. The pressure and temperature change can be observed and recorded by the digital display instrument. According to the change of the water level of the water tank and the gas flow meter in the pressure pump, the gas flow rate can be analyzed. The final content of gas hydrate, the formation of hydrate stability, and its related factors are the late stage of hydrate formation. The effect of the active agent on the formation of gas hydrate requires a comprehensive analysis of the results of the experiment.

3.1. Surface Active Agents for Gas Hydrate Formation. The effects of surfactants on the formation of natural gas hydrate can be divided into two types: promotion and inhibition. The effect of sodium sulfate (dodecyl) on the formation rate, the reaction time, the gas content rate of gas hydrate in the hydrate formation, and the temperature and pressure were studied.

SDS belongs to anionic surfactant. When the concentration of SDS increases, it will cause a sharp drop in the surface tension of solution. When the content of the surfactant in solution reaches the critical micelle concentration (CMC), the function of the surface active agent can be fully played [15]. Although CMC is influenced by temperature and pressure, the main factor of controlling the reaction is the degree of gas water contact in the process of hydrate formation. Under different reaction conditions, the optimum concentration may be different. Zhong and Rogers indicated that the concentration of the optimal reaction concentration and surface tension was not the same as that of induction time and surface tension [16]. As shown in Figure 2, when the concentration of SDS reached $280 \mathrm{ppm}$, the time of induction was stable. But the surface tension of the solution decreased obviously when the concentration of SDS reached $1100 \mathrm{ppm}$. Therefore, the optimum concentration of the active agent should be determined according to the reaction conditions, the volume of the reactor, and the mixed mode of gas and water.

Surface active agent has the function of solubilization, dispersion, and foaming. Therefore, the formation rate of gas hydrate in the surfactant system is obviously accelerated. 


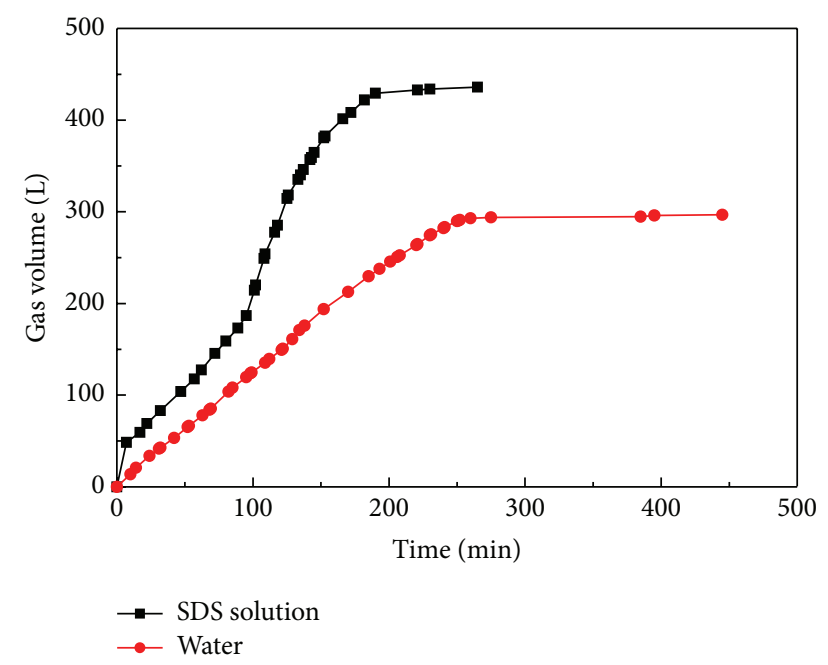

FIGURE 3: Gas consumption volume versus time for hydrate formation.

At $6 \mathrm{MPa}, 277.25 \mathrm{~K}$ conditions, it is found that the final gas content of hydrate formation in surfactant solutions has been significantly improved by spray atomization and on the concentration of $2.5 \mathrm{~L} 300$ ppm SDS solution and the condition of the same water for experiments, as shown in Figure 3. From Figure 3, it can be seen that the formation rate of gas hydrate in the solution of SDS is faster than that of pure water. At the beginning of the reaction, the reaction rate of the solution was significantly higher than that in pure water. The reaction time of pure water is nearly 3 hours longer than that of surfactant solution. In the late reaction stage, the water in the SDS aqueous solution is still capable of reacting with the gas contact due to the surface active agent. The hydrate formation rate still maintains a relatively fast state. But in pure water, the result is the opposite. So pure water system in the late period of reaction gas consumption changes very slowly. These directly lead to the increase of the total reaction time and the higher water content. The final gas content of the solution is $435.96 \mathrm{~L}$, while the water is only $296.64 \mathrm{~L}$.

The formation of gas liquid contact interface is usually the first in the gas hydrate formation. In the surfactant aqueous solution, due to the solubilization of surfactant, the surfactant micelles in aqueous solution have a large amount of gas dissolved in the surfactant micelles. Therefore, the formation of hydrate in the surfactant aqueous solution can not only occur at the gas liquid interface, but also in the aqueous solution. This reduces the time of hydrate formation and increases the growth rate [5]. In addition, Zhong et al. considered that the effect of surface active agents on the formation of hydrates is more likely to be adsorbed on the surface of the metal than the surface active agents. It replaces a layer of water film formed on the surface of the metal wall. Because of the low temperature near the wall of the reactor, the water film of the similar structure of the ice like structure continues to exist in the water phase. It can generate fast the crystal nucleus with the gas, thus increasing the hydrate formation rate. According to the experiment it is also found that SDS solution hydrate generated along the reactor wall

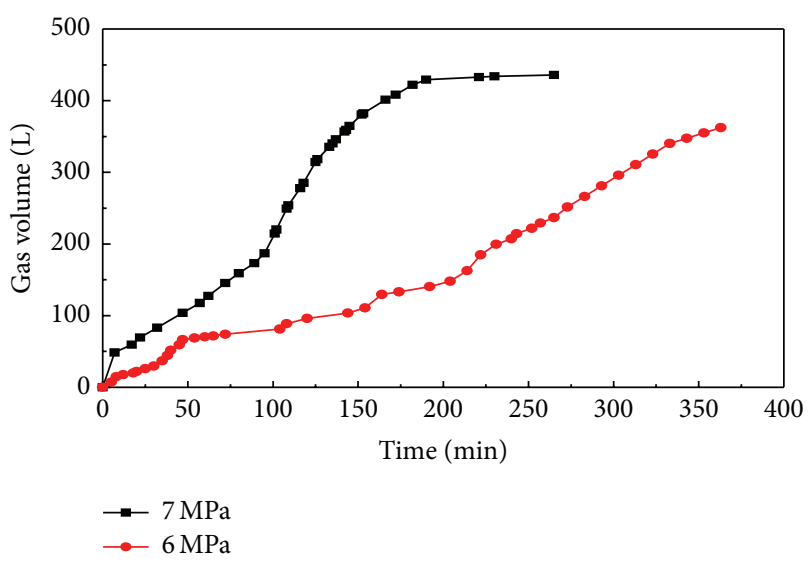

FIGURE 4: Different isobaric process of hydrate formation.

while hydrate form pure water is scattered at the bottom of the reactor. In macro characteristics, this explains the basic agreement with Zhong et al.

3.2. Temperatures and Pressures on Hydrate Production. The influence of temperature and pressure of gas hydrate are usually the thermodynamic condition of gas hydrate. They have important significance for the study of hydrate formation conditions. Formation of gas hydrate includes mass and heat transfer of gas molecules with water molecules. Undoubtedly, the mass and heat transfer conditions are the two main factors that affect the formation of gas hydrate. Temperature and pressure in the process of hydrate formation are very important [6].

By the classical theory of thermodynamics, the chemical potential difference of water in a two-phase system is pushing dynamic response. By the formula (1), the chemical potential difference is the single valued function of the pressure at a certain temperature. The higher the pressure, the greater the chemical potential difference and the stronger the reaction force. That is, the faster the reaction rate is obtained [7]:

$$
\vec{\nabla} \mu=R T \sum x_{i}\left(\frac{f_{i}^{\mathrm{eq}}}{f_{i}}\right) .
$$

In the formula, $R$ is gas constant; $T$ is reacting system temperature; and $f_{i}^{\text {eq }}$ and $f_{i}$ are the escape of the gas $i$ in the phase equilibrium pressure and the reaction pressure.

Experiments' result shows that the pressure has a direct effect on the reaction rate. The so-called overpressure which is the first reaction system pressure increases to a value higher than the reaction pressure and after some time falls back to system responses to stress. A distinct increase in the system's disturbance occurs, while the larger overpressure is more strongly disturbed. The longer the duration, the larger the system in the gas-liquid contact area. The faster the convection heat transfer, the higher the reaction rate. Figure 4 shows the change of gas volume consumption in the process of $7 \mathrm{MPa}$ and $6 \mathrm{MPa}$ and $277.25 \mathrm{~K}$ in the water. From the diagram it can be seen that the pressure increases not only to speed up the reaction rate, but also to increase the final gas 


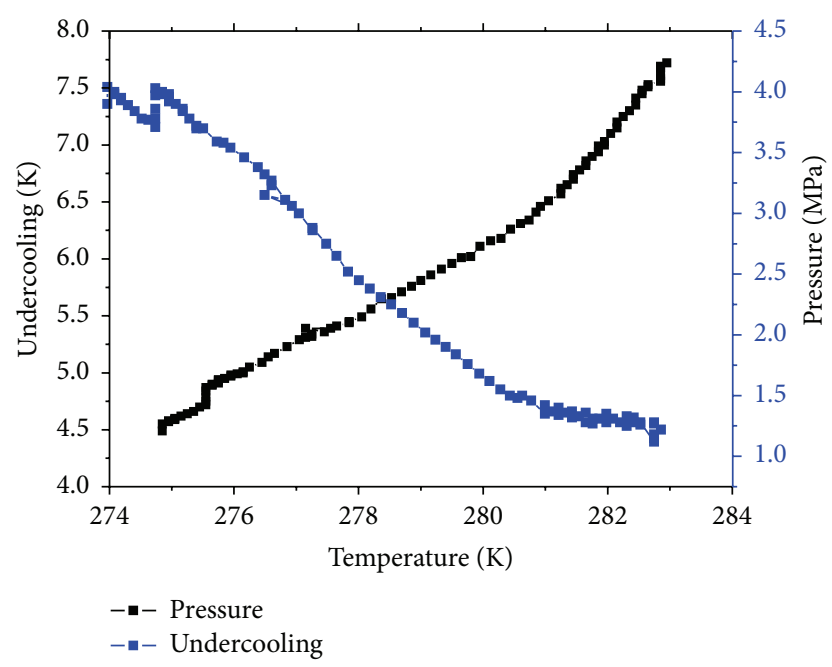

FIGURE 5: Pressure and undercooling versus temperature of hydrate formation.

content. For example, the final reaction time and gas content are $265 \mathrm{~min}$ and $435.96 \mathrm{~L}$ at $7 \mathrm{MPa}$, while they are $363 \mathrm{~min}$ 362.5 at $6 \mathrm{MPa}$.

The formation of hydrate is an exothermic process. The influence of temperature on the reaction process is not negligible. The reaction temperature not only affects the size of the equilibrium pressure, but also restricts the diffusion rate of the reaction. When the temperature increases, the reaction speed is faster and the reaction driving force is small. Figure 5 shows the temperature change of the hydrate formation in the spontaneous reaction of gas hydrate in the reaction conditions of the $14 \mathrm{~L}$ reaction. From the diagram, it is seen that the higher the temperature is, the smaller the temperature required for the reaction. But the corresponding equilibrium pressure increases. For example, the equilibrium pressure was $4.49 \mathrm{MPa}$ at $274.85 \mathrm{~K}$, and the undercooling is $3.9 \mathrm{~K}$. Under the same conditions, the equilibrium pressure is $7.72 \mathrm{MPa}$ at $282.95 \mathrm{~K}$, and the undercooling is $1.22 \mathrm{~K}$. Therefore, in the actual situation, temperature and pressure on the formation of gas hydrate should be taken into account to get the best match.

\section{Conclusions}

The hydrate formation process is a complex physical and chemical process. The surface active agent can obviously accelerate the reaction rate and increase the gas holdup of gas hydrate. Temperature and pressure have limited the formation of hydrate, and the best value should be sought for the second. A variety of external force fields (electric field, magnetic field, radiation field, and ultrasonic field) on hydrate nucleation formation have effect, but the research is still not deep enough. So further exploration is expected in the future.

\section{Conflict of Interests}

The authors declare that there is no conflict of interests regarding the publication of this paper.

\section{Acknowledgments}

The financial support was received from the Natural Science Foundation of China (nos. 51074111 and 51204120) and the Natural Science Foundation of Shanxi Province in China (no. 2011011005).

\section{References}

[1] E. D. Sloan and C. A. Koh, Clathrate Hydrates of Natural Gases, CRC Press; Taylor \& Francis Group, New York, NY, USA, 3rd edition, 2008.

[2] A. Eslamimanesh, A. H. Mohammadi, D. Richon, P. Naidoo, and D. Ramjugernath, "Application of gas hydrate formation in separation processes: a review of experimental studies," Journal of Chemical Thermodynamics, vol. 46, no. 2, pp. 62-71, 2012.

[3] B. Zhang and Q. Wu, "Thermodynamic promotion of tetrahydrofuran on methane separation from low-concentration coal mine methane based on hydrate," Energy \& Fuels, vol. 24, no. 4, pp. 2530-2535, 2010.

[4] X.-S. Li, J. Cai, Z.-Y. Chen, and C.-G. Xu, "Hydrate-based methane separation from the drainage coal-bed methane with tetrahydrofuran solution in the presence of sodium dodecyl sulfate," Energy and Fuels, vol. 26, no. 2, pp. 1144-1151, 2012.

[5] D.-L. Zhong, K. Ding, J. Yan, C. Yang, and D.-J. Sun, "Influence of cyclopentane and SDS on methane separation from coal mine gas by hydrate crystallization," Energy and Fuels, vol. 27, no. 12, pp. 7252-7258, 2013.

[6] J. Zhao, Y. Tian, and Y. Zhao, "Separation of methane from coal bed gas via hydrate formation in the presence of tetrahydrofuran and sodium dodecyl sulfate," Chemistry and Technology of Fuels and Oils, vol. 49, no. 3, pp. 251-258, 2013.

[7] L. C. Ho, P. Babu, R. Kumar, and P. Linga, "HBGS (hydrate based gas separation) process for carbon dioxide capture employing an unstirred reactor with cyclopentane," Energy, vol. 63, pp. 252-259, 2013.

[8] M. D. Jager and E. D. Sloan, "The effect of pressure on methane hydration in pure water and sodium chloride solutions," Fluid Phase Equilibria, vol. 185, no. 1-2, pp. 89-99, 2001.

[9] B. ZareNezhada, M. Mottahedina, and F. Varaminiana, "Experimental and theoretical investigations on the enhancement of methane gas hydrate formation rate by using the kinetic additives," Petroleum Science and Technology, vol. 33, no. 8, pp. 857-864, 2015.

[10] K. Okutani, Y. Kuwabara, and Y. H. Mori, "Surfactant effects on hydrate formation in an unstirred gas/liquid system: an experimental study using methane and sodium alkyl sulfates," Chemical Engineering Science, vol. 63, no. 1, pp. 183-194, 2008.

[11] R. Ohmura, S. Kashiwazaki, S. Shiota, H. Tsuji, and Y. H. Mori, "Structure-I and structure-H hydrate formation using water spraying," Energy and Fuels, vol. 16, no. 5, pp. 1141-1147, 2002.

[12] J.-Y. Liu, J. Zhang, Y.-L. Liu, X.-H. Tan, and J. Zhang, "Experimental and modeling studies on the prediction of gas hydrate formation," Journal of Chemistry, vol. 2015, Article ID 198176, 5 pages, 2015.

[13] S. Khosharay, H. Roosta, and F. Varaminian, "Investigation on the kinetics of methane and carbon dioxide hydrates by using a modified kinetic model," Journal of Natural Gas Science and Engineering, vol. 26, pp. 587-594, 2015.

[14] J. M. Lee, S. J. Cho, J. D. Lee, P. Linga, K. C. Kang, and J. Lee, "Insights into the kinetics of methane hydrate formation 
in a stirred tank reactor by in situ raman spectroscopy," Energy Technology, vol. 3, no. 9, pp. 925-934, 2015.

[15] N. Gnanendran and R. Amin, "Modelling hydrate formation kinetics of a hydrate promoter-water-natural gas system in a semi-batch spray reactor," Chemical Engineering Science, vol. 59, no. 18, pp. 3849-3863, 2004.

[16] Y. Zhong and R. E. Rogers, "Surfactant effects on gas hydrate formation," Chemical Engineering Science, vol. 55, no. 19, pp. 4175-4187, 2000. 

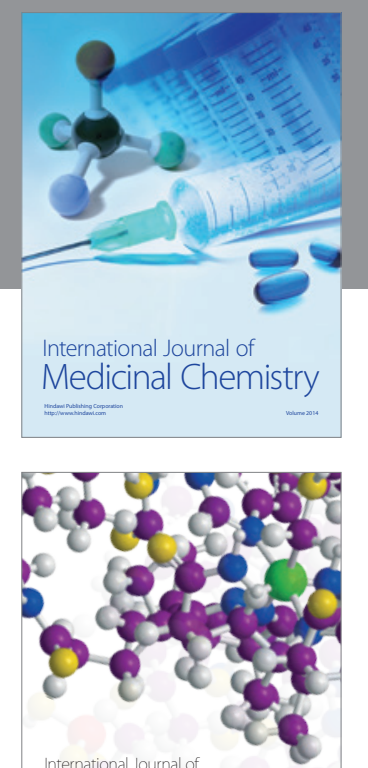

\section{Carbohydrate} Chemistry

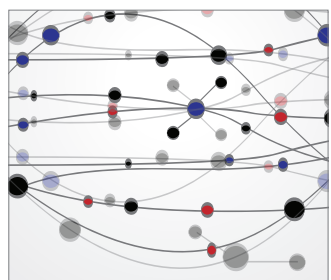

The Scientific World Journal
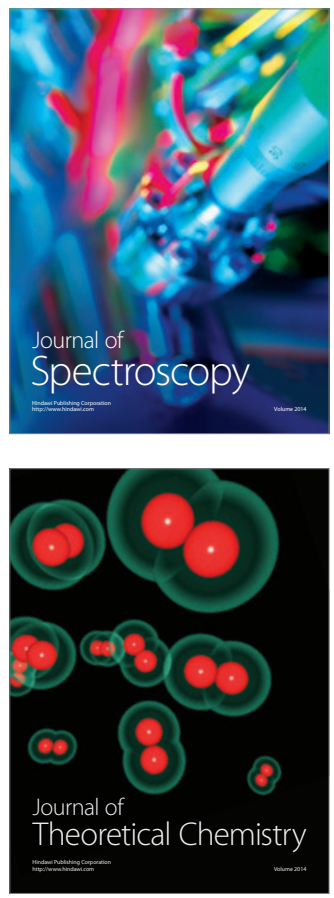
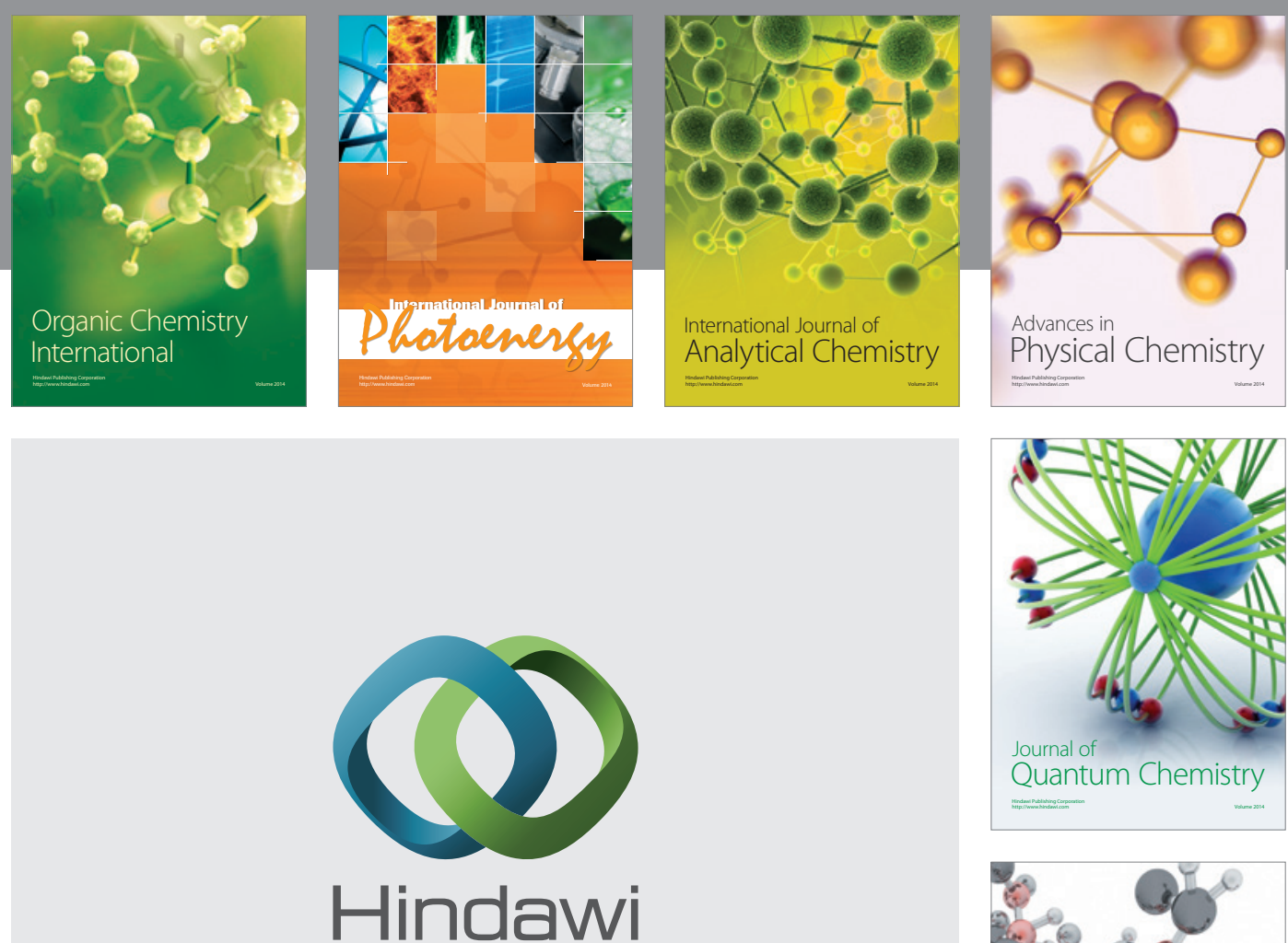

Submit your manuscripts at

http://www.hindawi.com

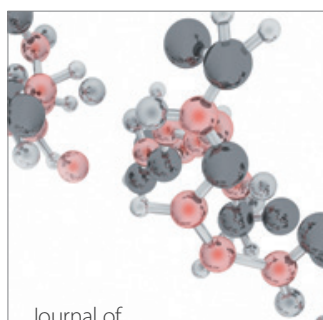

Analytical Methods

in Chemistry

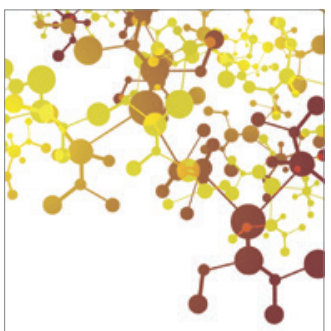

Journal of

Applied Chemistry

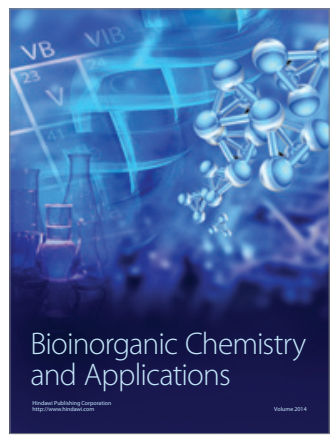

Inorganic Chemistry
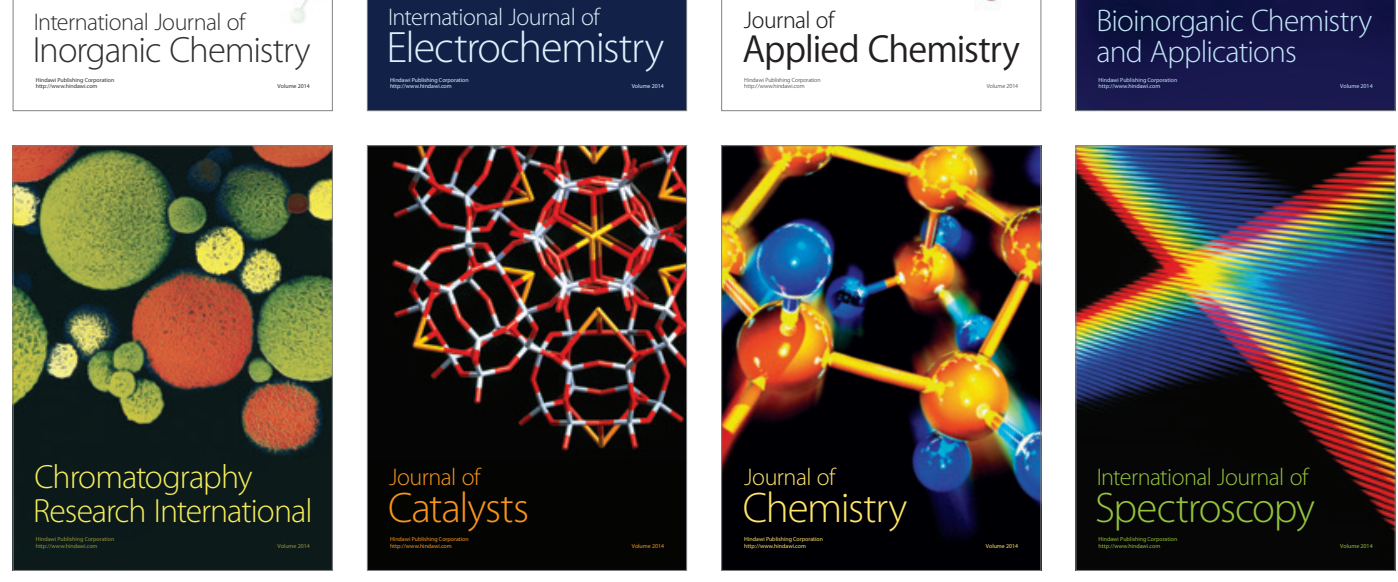